\title{
Insight into potential mechanisms of hypobaric hypoxia-induced learning and memory deficit - Lessons from rat studies
}

Human and Experimental Toxicology (C) The Author(s) 2017 Reprints and permission: sagepub.co.uk/journalsPermissions.nav DOI: $10.1 \mid 77 / 0960327$ | $166897 \mid 4$ journals.sagepub.com/home/het

(A) SAGE

\section{EYA Qaid', R Zakaria', SF Sulaiman' ${ }^{2}$, NA Mohd Yusof ${ }^{3}$, N Shafin', Z Othman', AH Ahmad' and CB Abd Aziz'}

\begin{abstract}
Impairment of memory is one of the most frequently reported symptoms during sudden hypoxia exposure in human. Cortical atrophy has been linked to the impaired memory function and is suggested to occur with chronic high-altitude exposure. However, the precise molecular mechanism(s) of hypoxia-induced memory impairment remains an enigma. In this work, we review hypoxia-induced learning and memory deficit in human and rat studies. Based on data from rat studies using different protocols of continuous hypoxia, we try to elicit potential mechanisms of hypobaric hypoxia-induced memory deficit.
\end{abstract}

\section{Keywords}

High altitude, hypoxia, learning, memory, rats

\section{Introduction}

Hypoxia is defined as inadequate oxygen supply to the cells and tissues of the body. In this review, the term 'hypoxia' refers to 'hypoxic hypoxia', which is also the most common type of hypoxia and is due to low arterial oxygen concentration or hypoxemia. Low arterial oxygen concentration is first detected by sensory receptors in the carotid or aortic body and impulses are sent to cardiorespiratory centre in the medulla. ${ }^{1}$ The body responds to normalize the decrease in the oxygen arterial concentration by activation of the sympathetic nervous system which leads to increased heart rate, blood pressure, ventilation and the production of stress hormones. ${ }^{2-4}$ However, in the case of very low arterial oxygen concentration, the body will fail to normalize.

Hypoxia has long been known to diminish brain function in humans and animals. ${ }^{5-7}$ In humans, the degree of hypoxia required to impair performance, and the task most sensitive to it, is controversial. ${ }^{7}$ It has been reported that the earliest cognitive signs and symptoms of hypoxia are typically experienced around $15-16 \%$ of oxygen concentration, ${ }^{8}$ and complex information processing and learning are among the most sensitive processes to be affected. ${ }^{9}$
Impairment of memory is one of the most frequently reported symptoms during sudden hypoxia exposure, for instance, during hypoxia-awareness training of aircrews or after an in-flight hypoxic incident. ${ }^{10}$ However, the effects of acute hypoxia on memory have only been sporadically studied in laboratory-controlled conditions, mainly for military aviation $^{10}$ and even less for civilian aviation. With more severe hypoxia, critical judgement declines, and this proceeds to stupor, coma and, finally, death if the hypoxia is not reversed. ${ }^{11}$

'Department of Physiology, School of Medical Sciences, Universiti Sains Malaysia, Kubang Kerian, Malaysia

${ }^{2}$ School of Biological Sciences, Universiti Sains Malaysia, Penang, Malaysia

${ }^{3}$ Department of Anatomy, School of Medical Sciences, Universiti Sains Malaysia, Kubang Kerian, Malaysia

${ }^{4}$ Department of Psychiatry, School of Medical Sciences, Universiti Sains Malaysia, Kubang Kerian, Malaysia

\section{Corresponding author:}

R Zakaria, Department of Physiology, School of Medical Sciences, Universiti Sains Malaysia, 16150 Kubang Kerian, Malaysia.

Email: rahimah@usm.my 


\section{Materials and methods}

All articles indexed to MEDLINE and books were searched using the following key words: hypoxia, hypobaric, normobaric, learning and memory. This review mainly aims to look into how different protocols of continuous hypoxia induced by hypobaric/normobaric hypoxic chamber affect the learning and memory of adult rats. Results from brain hypoxia induced by invasive procedures such as ligation of arterial supply to the brain ${ }^{12}$ or by chemical induction such as carbon monoxide hypoxia ${ }^{13}$ are not included. The effects of different hypoxia protocols on learning and memory in rats are summarized in Table 1.

\section{Hypoxia and memory impairment in human}

Studies on the effects of hypoxia in life sciences are generally performed either by decreasing barometric pressure (PB), leading to hypobaric hypoxia as shown by Bert, ${ }^{39}$ or by decreasing oxygen fraction $\left(\mathrm{F}_{\mathrm{I}} \mathrm{O}_{2}\right)$ without changing $\mathrm{PB}$ as shown by Barcroft ${ }^{40}$ in his 'Glass House' experiment (normobaric hypoxia (NH)). It has been shown that exposure to hypobaric hypoxia $\left(\mathrm{F}_{\mathrm{I}} \mathrm{O}_{2}<20.9 \%\right.$; $\left.\mathrm{PB}<760 \mathrm{mmHg}\right)$ and $\mathrm{NH}$ $\left(\mathrm{F}_{\mathrm{I}} \mathrm{O}_{2}<20.9 \%\right.$; $\left.\mathrm{PB}=760 \mathrm{mmHg}\right)$ may or may not result in different physiological responses in human subjects. $^{41-43}$

In a study by Shukitt-Hale et al., ${ }^{44} 23$ nonacclimatized males were exposed to 500,4200 or $4700 \mathrm{~m}$ in an altitude chamber for 4-5 h. The study demonstrated that the higher the altitude and the longer the duration of exposure, the more severe mood and memory impairment. A similar study was later conducted by Du et al. ${ }^{45}$ involving 18 healthy young male subjects exposed to $300 \mathrm{~m}$ (control group), 2800, 3600 and $4400 \mathrm{~m}$ altitude in hypobaric chamber. Compared to control group, after exposure to $2800 \mathrm{~m}$ for $1 \mathrm{~h}$, only the performance of continuous recognition memory decreased significantly. After exposure to $3600 \mathrm{~m}$, total reaction time in all tests increased significantly and performance decreased, but the error rates in memory scanning and space memory test were unchanged. During exposure to $4400 \mathrm{~m}$, performance of memory test decreased further and error rates also increased significantly. It was concluded that the performance of human short-term memory deteriorates after exposure to acute mild and moderate hypobaric hypoxia for $1 \mathrm{~h}$, and these effects are aggravated with the increase in altitude as seen in another study. ${ }^{46}$ In a study by Malle et al. ${ }^{10}$ involving a bigger number of participants, 28 subjects in the experimental group were exposed to a simulated altitude level of $10,000 \mathrm{~m}(31,000 \mathrm{ft})$ in a hypobaric chamber, while 29 subjects in the control group stayed at sea level. The short-term (working) memory assessed using paced auditory serial addition test (PASAT) showed that performance was strongly impaired in the hypoxic group with increased mean error frequency rate. While working memory performance decreased linearly with hypoxemia, peripheral oxygen saturation $\left(\mathrm{SpO}_{2}\right)$ was found to be a weak predictor of PASAT performance and vice versa.

Structural brain alterations following exposure to high altitude may include brain swelling, ${ }^{47}$ increase in the number of white matter hyperintensities ${ }^{48}$ and grey and white matter atrophy, ${ }^{49-51}$ contributing to a reduction in total brain volume. Hemosiderin deposits (microhaemorrhages) have also been reported in subjects who have experienced high-altitude cerebral edema. ${ }^{52}$ Cortical atrophy is linked to impaired cognitive function and has been suggested to occur with chronic high-altitude exposure. ${ }^{53}$ However, human studies shed little light on the precise molecular mechanism(s) of hypoxia-induced memory impairment.

\section{Animal studies related to hypoxia}

It has been established that the brain is highly sensitive to hypoxia and that some areas, such as the hippocampus, are especially vulnerable to hypoxic damage. ${ }^{54}$ Compared to human brain, the brain of smaller animal (such as rat) is more resistant to hypoxia because of its higher capillary density, that is, much more severe hypoxic condition must be applied to overtly damage its brain tissues relative to human. ${ }^{17}$ Male rats are more susceptible to hypoxia compared to female rats; hence, most hypobaric hypoxia studies were conducted on male rats to obtain better and more conclusive results. ${ }^{55-56}$

Depending on the objectives of the study, smaller animals are either exposed to continuous or intermittent hypoxia. Continuous hypoxia with or without low PB rat model is commonly used to study the effect of acute mountain sickness, diseases associated with limited oxygen supply to the brain such as chronic obstructive pulmonary disease and acute respiratory distress syndrome, medication and drugs, concussion or changes in air quality, for example, in a nuclear power plant. Intermittent hypoxia rat model, on the other hand, is commonly used to study the effects of 


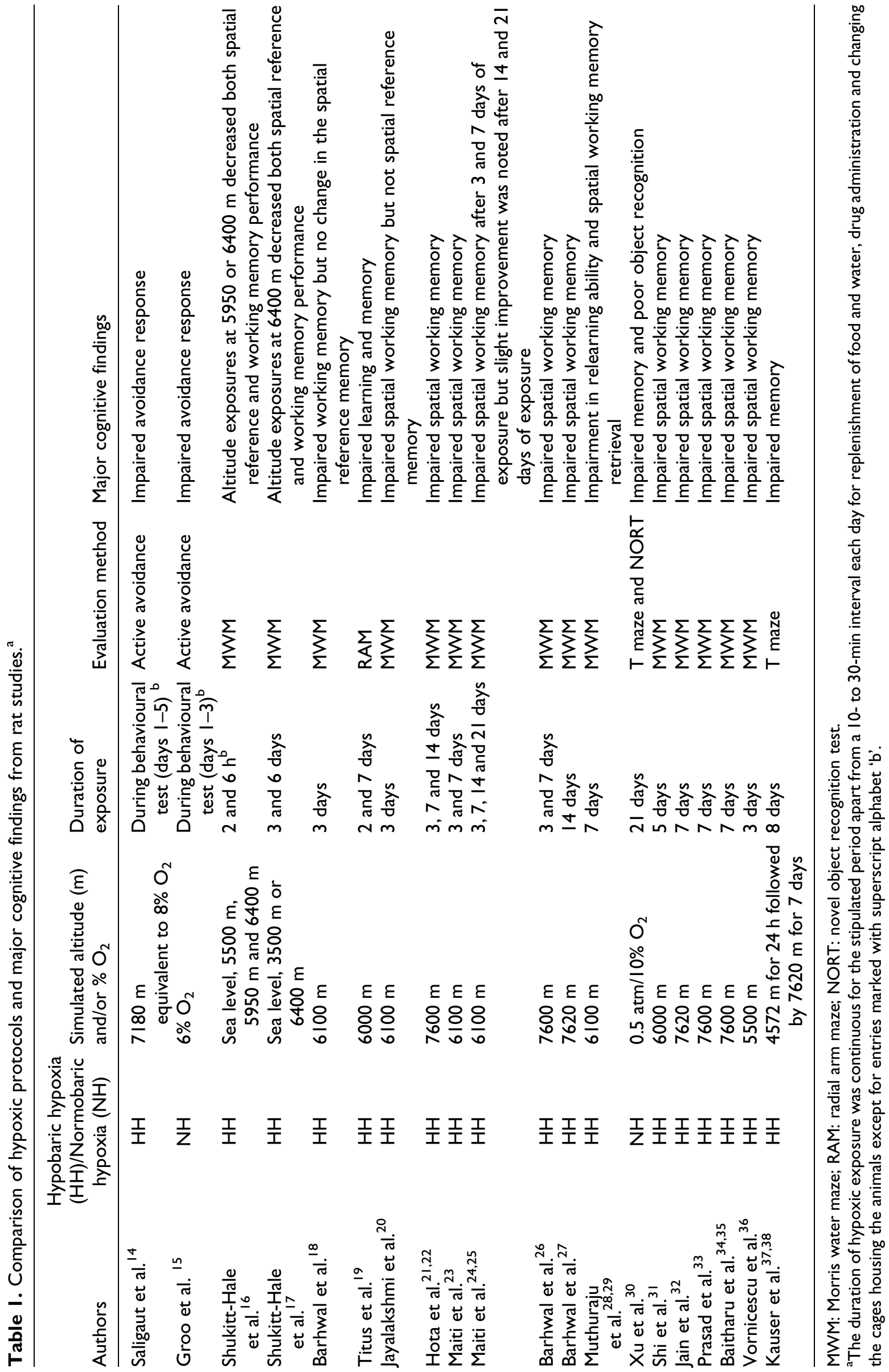


obstructive sleep apnoea. Furthermore, understanding how hypoxia alters brain function has implications for understanding other metabolic encephalopathies as well as aging and age-related disorders, such as Alzheimer's disease. ${ }^{57}$

\section{Hypoxia and memory impairment in rats}

Groo et al. ${ }^{15}$ used spontaneously hypertensive rats (SHRs) to study avoidance response to hypoxia as they were reported to be more sensitive to stressful stimuli ${ }^{58,59}$ and showed greater hypoxic vulnerability than normotensive Wistar rats. The SHRs were exposed to either $\mathrm{NH}$ ( $6 \%$ oxygen) or normoxic conditions. Lowering the oxygen contents of inspired air to $6 \%$ impaired acquisition of the avoidance response, and the difference between the performance (in percentage of conditioned avoidance response) of animals kept under normoxic and hypoxic conditions was significant on day 3 (at $69.2 \%$ and $38.0 \%$, respectively). Hypoxia was also found to increase the incidence of escape failures. These findings are in agreement with earlier study findings by Saligaut et al., ${ }^{14}$ where the acquisition of a conditioned avoidance response was also impaired in hypobaric hypoxia at 300 Torr $(7180 \mathrm{~m}$; equivalent to $8 \%$ oxygen content).

\section{Hippocampal damage}

Direct correlation between working memory impairment and level of altitude was evidenced in a study involving Fischer male rats exposed to various altitudes equivalent to sea levels 5500,5950 and $6400 \mathrm{~m}$, for 2 and $6 \mathrm{~h}^{16}$ In a later study by the same group of researchers, two groups of rats were exposed to $6400 \mathrm{~m}$ altitude for either 72 or $144 \mathrm{~h}$. It was found that the longer the time of exposure, the more noticeable the hippocampal damage (delayed neurotoxicity) as shown by $78 \%$ neuronal damage after $144 \mathrm{~h}$ compared to $50 \%$ after $72 \mathrm{~h}$ of exposure. ${ }^{53}$ Titus et al. ${ }^{19}$ showed that hippocampal-dependent spatial learning in rats was affected marginally following 2 days of exposure to simulated hypobaric hypoxia at $6000 \mathrm{~m}$, while 7 days of exposure severely affected learning of partially baited radial-arm maze (RAM) task. The study also found that exposure for 2 days to hypobaric hypoxia resulted in minimal deleterious effects on the CA1 pyramidal neurons, while exposure for 7 days caused a significant decrease in the number of branching points, intersections and dendritic length. Unlike the CA1 pyramidal neurons, CA3 neurons exhibited dendritic atrophy following both 2 and 7 days of hypobaric hypoxia exposure. Thus, CA3 neurons are more vulnerable to hypoxic insult compared to CA1 neurons. ${ }^{23,60,61}$ Findings by Titus et al. are in agreement with the observation of neuronal degeneration in rat hippocampus exposed to hypobaric hypoxia in previous studies ${ }^{23,53}$ as well as a later study by Prasad et al. ${ }^{33}$

Maiti et al. ${ }^{24}$ investigated spatial memory functions and dendritic changes in CA1, CA3 and entorhinal cortex of hippocampus, and layer II of prefrontal cortex (PFC) in rats exposed to simulated hypobaric hypoxia at $6100 \mathrm{~m}$, but with different durations of hypobaric hypoxia exposure, that is, 3, 7, 14 and 21 days. There was impairment of spatial memory after 3 and 7 days, but slight improvement of spatial memory was noted after 14 and 21 days of exposure. The study suggested that hypobaric hypoxia induces dendritic plasticity of PFC and hippocampal pyramidal neurons of rat brain, which may be associated with improvement of spatial memory function after 21 days of hypobaric hypoxia exposure. These findings are in contrast with a recently published data on the adverse effects of hypobaric hypoxia on the brain which became more severe after 4 weeks compared to 2 days of exposure. The total brain weight and oxidative stress in all three brain regions (striatum, hippocampus and cortex) were significantly increased after 4 weeks compared to after 2 days of hypobaric hypoxia. The study, however, did not assess cognitive function. ${ }^{62}$ Further studies are necessary to attest to the possibility of compensatory stage after 2-3 weeks of hypobaric hypoxia exposure followed by a decompensated stage after 4 weeks.

\section{Brain oxidative stress}

Liu et al. ${ }^{63}$ reported that oxidative damage to hippocampus impairs spatial memory of rats. Rats showed impairment of working memory but no change in reference memory after 3 days of exposure to simulated hypobaric hypoxia at $6100 \mathrm{~m} \cdot{ }^{18,20}$ The study revealed a significant decrease in reduced glutathione (GSH) levels with concomitant increase in oxidized glutathione (GSSG) in the hypoxic rats. The increased generation of free radicals might have resulted in increased utilization of $\mathrm{GSH}$, thus leading to increased GSSG synthesis. The increase in GSH utilization is further accompanied by decreased GSH 
synthesis as evidenced by decreased GSH reductase activity, thus depleting antioxidant status. This might have been triggered through low levels of NADPH, which is a cofactor for GSH reductase to convert GSSG to GSH. Increased generation of free radicals during hypobaric hypoxia is due to low oxygen availability, and this may be explained by leakage of free electrons triggering a chain reaction resulting in the formation of hydrogen peroxide and reactive hydroxyl radicals. ${ }^{64}$ These reactive oxygen species (ROS) have a high affinity for membrane lipids, ${ }^{65}$ especially in the brain, as it is rich in polyunsaturated fatty acids. The ROS leads to lipid peroxidation and membrane damage. ${ }^{66}$ In addition, there is deterioration in the antioxidant defence mechanism that under normal physiological conditions scavenges the free radicals produced in the cell as by-products of various metabolic pathways. Similar findings were noted by Hota et al. ${ }^{21,22}$ in studies on hypobaric hypoxia and oxidative stress. In these studies, however, acclimatization to oxidative stress occurred after prolonged hypobaric hypoxia exposure, that is, 14 days. The study also pointed towards the crucial role that glutamate might play in causing the hypobaric hypoxia-induced oxidative stress. Since numerous biochemical and molecular pathways are involved in the stress response of the cells, the precise mechanism responsible for the onset of acclimatization to oxidative stress still remains to be elucidated.

A study by Shi et al. ${ }^{31}$ supported earlier findings on the oxidative stress and apoptosis associated with memory impairment in rats exposed to hypobaric hypoxia. The study showed that exposure to simulated hypobaric hypoxia at $6000 \mathrm{~m}$ for 5 days caused spatial memory impairment as well as oxidative stress (increased lactate dehydrogenase activity, decreased GSH level, decreased superoxide dismutase (SOD) level, inhibition of GSH synthesis and greater utilization of GSH for detoxification of hypoxia-induced free radicals, thus leading to increased GSSG synthesis) and apoptosis in different regions of the brain in rats. They also found that the hippocampus is more susceptible to hypoxia when compared with the cortex.

A later study by Baitharu et al. ${ }^{34,35}$ found that hypobaric hypoxia-induced memory impairment was associated with neurodegeneration along with alteration in nitric oxide (NO), glucocorticoid, corticosterone, oxidative stress and acetylcholinesterase (AChE) activity in the hippocampal region. In this study, rats were exposed to a simulated altitude of $7600 \mathrm{~m}$ in a specially designed animal decompression chamber for 7 days. Another study showed that a shorter and lower simulated altitude exposure also resulted in spatial memory impairment. ${ }^{36}$ Exposure of rats to an altitude of $5500 \mathrm{~m}(375 \mathrm{mmHg})$ for 3 days was found to induce oxidative stress as evidenced by significant increase in malondialdehyde and reduction in GSH in serum and brain tissue, and neuronal death associated with reactive astrogliosis in hippocampus and superjacent cortex.

\section{Signalling pathways involved in oxidative stress}

Barhwal et al. ${ }^{26,27}$ examined the signalling cascades involved in mediating oxidative stress neuronal damage in hypobaric hypoxia. The study revealed increased thioredoxin (Trx-1) expression and increased extracellular-signal-regulated kinase (ERK) phosphorylation in rats exposed to hypobaric hypoxia for 14 days. ${ }^{67}$ Trx-1 increases hypoxia-inducible factor- $1 \alpha$ (HIF-1 $\alpha)$ expression in both normoxic and hypoxic conditions. ${ }^{68}$ The HIF- $1 \alpha$ activates many genes involved in erythropoiesis, angiogenesis, energy metabolism, proliferation/cell survival, apoptosis and generation of NO. ${ }^{69-71}$ Alteration of NO production plays an important role in brain injury in conditions of hypoxia/reoxygenation. ${ }^{67} \mathrm{NO}$ produced in excess in the brain under the action of hypoxiainduced neuronal nitric oxide synthases rapidly reacts with superoxide anion to form peroxynitrite $\left(\mathrm{ONOO}^{-}\right)$, a more toxic metabolite that causes brain injury. ${ }^{72}$ The peroxynitrite is responsible for protein denaturation, lipid peroxidation, DNA damage and depletion of antioxidant defences. ${ }^{25}$

On the other hand, ERK pathway contributes to nuclear factor (erythroid-derived 2)-like 2 (Nrf2) protein stabilization and may result in the increased translocation of Nrf2 into the nucleus. ${ }^{73} \mathrm{Nrf} 2$ is the central transcription factor involved in regulating the expression of antioxidant enzymes like GSH S-transferase and SOD that are important in protecting the cells against oxidative damage. However, despite increased nuclear translocation of Nrf2, there is increased free radical generation, protein oxidation and lipid peroxidation in hypobaric hypoxia rats. ${ }^{27}$

\section{Calcium overload-induced oxidative stress}

Calcium overload in the neurons is previously reported to generate ROS by activating phospholipase A2, xanthine oxidase and monoamine oxidase. Release of cytochrome c from the mitochondria due 
to calcium sequesteration is also known to generate ROS and trigger apoptotic cell death. ${ }^{26}$

The elevation of intracellular calcium concentrations and its L-type calcium channel expression, as well as increased calpain expression, have been demonstrated in rats exposed to hypobaric hypoxia. ${ }^{26}$ Calpain and L-type calcium channel expressions are maximal on day 7 of hypobaric hypoxia exposure and decline on day 14. Calcium is known to activate calpain that mediates proteolysis of selective proteins and significantly contributes to neuronal damage ${ }^{74}$ in several hypoxic and ischemic models. ${ }^{75,76}$ Calcium is also known to be sequestered into the mitochondria in excitotoxic conditions, thus resulting in release of cytochrome $\mathrm{c}$ that triggers mitochondria-mediated apoptotic cascades. ${ }^{77,78}$ Increased cytosolic cytochrome $\mathrm{c}$ on day 7 is also demonstrated followed by a marginal decline on day 14 of hypoxic exposure. Expression of active caspase 3, however, shows a different trend with its maximal level on day 14 of hypoxic exposure. This is, however, in accordance with another previous study that reported progressive increase in caspase 3 activity and neurodegeneration with increased duration of exposure. ${ }^{33}$ This anomaly in caspase 3 activity may be due to existence of other signalling cascades on day 14 of chronic hypoxic exposure $^{21}$ and warrants further investigation.

\section{Glutamate excitotoxicity}

The synthesis and release of neurotransmitters are particularly sensitive to hypoxia. Hypobaric hypoxia induces increased release of excitatory amino acid such as glutamate ${ }^{79}$ and upregulation of $N$-methyl-Daspartate (NMDA) receptors. In a study by Hota et al., ${ }^{80}$ male Sprague Dawley rats were exposed to simulated hypobaric hypoxia at $7600 \mathrm{~m}$ for 3, 7 and 14 days. Memory impairment in rats was maximal after 14 days of hypobaric hypoxia exposure. It was associated with significant increase in expression of NR1 subunit of NMDA receptor, indicating overstimulation of the NMDA receptor by hypobaric hypoxia. Binding of glutamate to NMDA receptors in hypoxic ischemia has also been reported to cause free radical production and robust influx of calcium ions into the cytosol leading to calcium overload. ${ }^{81}$ This excess calcium is sequestered by the mitochondria through the calcium uniporter in the inner mitochondrial membrane at the expense of mitochondrial membrane potential. ${ }^{82-86}$ This in turn causes generation of ROS by the mitochondria that may lead to mitochondrial membrane damage and may cause the release of cytochrome $\mathrm{c}$ from the mitochondria during hypoxia. ${ }^{87,88}$ It is well known that cytochrome $\mathrm{c}$ is released from the mitochondria to the cytoplasm ${ }^{89}$ as an outcome of mitochondrial dysfunction that may trigger apoptosis. This activates a plethora of proapoptotic proteins such as Bax, caspase-3, Smac/Diablo, Bid, Bad, Apaf-1 and many others. ${ }^{90,91}$

\section{Alteration in cholinergic and adrenergic systems}

Apart from the crucial role of oxidative stress and glutamate excitotoxicity in mediating cognitive deficits following exposure to hypobaric hypoxia, cholinergic systems are also known to be involved. ${ }^{92}$ Previous reports have discussed that decreased choline acetyltransferase, ${ }^{93}$ increased $\mathrm{AChE}$ and decreased $\alpha 7$ nicotinic and muscarinic M1 acetylcholine $(\mathrm{ACh})$ receptors ${ }^{94}$ are closely associated with cognitive deficits. Muthuraju et al. ${ }^{28,29}$ revealed that impairment in relearning ability and memory retrieval in rats exposed to hypobaric hypoxia was associated with decreased $\mathrm{ACh}$ and increased $\mathrm{AChE}$ levels which then led to morphological damage in cortical and hippocampal neurons. Administration of AChE inhibitors, such as physostigmine and galantamine, resulted in amelioration of the hypobaric hypoxia-induced neuronal morphological damage in cortex and hippocampus. These AChE inhibitors improved ACh level, decreased $\mathrm{AChE}$ activity and increased ACh synthesis by increasing acetyltransferase activity. ${ }^{95}$

The greatest amount of nerve growth factor (NGF) is produced in the cortex, hippocampus and pituitary gland, although significant quantities are also produced in other areas, including the basal ganglia, thalamus, spinal cord and in the retina. ${ }^{96}$ The NGF plays a pivotal role in the survival and function of cholinergic neurons of the basal forebrain complex ${ }^{97}$; such functions include attention, arousal, motivation, memory and consciousness.

Other studies associate memory impairment with adrenergic dysregulation and neuronal damage in medial PFC. In these studies, rats were habituated at simulated altitude of $4572 \mathrm{~m}$ for 1 day followed by exposure to simulated altitude of $7620 \mathrm{~m}$ for a further 7 days. ${ }^{37,38}$ It was suggested that norepinephrine (NE) dysregulation under hypobaric hypoxia might have been one of the possible underlying mechanisms leading to cognitive deficits and associated morphological 
damage. This is due to the fact that working memory is regulated by the PFC and is created by networks of PFC neurons engaged in recurrent excitation generating persistent activity. ${ }^{98}$ If the PFC recurrent excitatory firing is profoundly altered by the $\mathrm{NE}$ dysfunction, working memory functions will also be affected. $^{98,99}$

\section{Brain-derived neurotrophic factor}

Jain et al. ${ }^{32}$ examined the cellular and molecular pathways related to hypobaric hypoxia-induced neuronal cell death. Adult male Sprague Dawley rats exposed to hypobaric hypoxia equivalent to $7620 \mathrm{~m}$ for 7 days resulted in spatial memory impairment and neurodegeneration that was related to low brain-derived neurotrophic factor (BDNF). The low BDNF inhibits $\mathrm{PI} 3 \mathrm{~K} / \mathrm{AKT}$ pathway resulting in activation of GSK $3 \beta$ and caspase 3 which further augments neuronal apoptosis and memory impairment.

cAMP response element binding protein (CREB) is a key transcription factor involved in several critical functions of the brain including learning, neuronal plasticity and cell survival. ${ }^{100} \mathrm{CREB}$ has been shown to be the key mediator for BDNF-mediated cell survival as studies showed that silencing the transcriptional activity of CREB impaired BDNF protection. ${ }^{101}$ CREB can be activated by various kinases including ERK, AKT and GSK3 $\beta .^{102}$

Although there was increased CREB phosphorylation in hypoxia as observed from the total CREB to phospho CREB ratio, total CREB was decreased when compared to the normoxic group, thus resulting in its decreased availability for phosphorylation. The decreased CREB expression in hypobaric hypoxia is probably due to the excitotoxic neuronal loss and free radical-mediated protein degradation as indicated by increased protein carbonyls. ${ }^{22}$

\section{Conclusion}

Hypobaric hypoxia studies conducted in rats provide some insight into the underlying mechanism of hypobaric hypoxia-induced memory loss. Exposure to hypobaric hypoxia in rats induces glutamate excitotoxicity and increases influx of calcium ion by NR1 subunit of NMDA receptor and L-type calcium channel upregulation. ${ }^{21,22,26}$ It also induces oxidative stress $^{18,20-22,34}$ via apoptotic signalling pathways leading to hippocampal cell apoptosis. ${ }^{26,27,32}$ Hypobaric hypoxia exposure also impairs cholinergic ${ }^{28,29}$ and adrenergic ${ }^{37,38}$ systems and lowers BDNF level which are important for memory function. All of these mechanisms may be related to learning and memory deficit in human exposed to hypobaric hypoxia.

\section{Declaration of Conflicting Interests}

The author(s) declared no potential conflicts of interest with respect to the research, authorship, and/or publication of this article.

\section{Funding}

The author(s) disclosed receipt of the following financial support for the research, authorship, and/or publication of this article: This research received funding from USM Short-term Grant (304/PPSP/61313086).

\section{References}

1. Kalia M and Welles RV. Brain stem projections of the aortic nerve in the cat: a study using tetramethyl benzidine as the substrate for horseradish peroxidase. Brain Res 1980; 188: 23-32.

2. Fishman AP, Fritts HW and Cournand A. Effects of acute hypoxia and exercise on the pulmonary circulation. Circulation 1960; 22: 204-215.

3. Buchheit M, Richard R, Doutreleau S, et al. Effect of acute hypoxia on heart rate variability at rest and during exercise. Int J Sports Med 2004; 25: 264-269.

4. Richalet JP, Letournel M and Souberbielle JC. Effects of high-altitude hypoxia on the hormonal response to hypothalamic factors. Am J Physiol 2010; 299: 1685-1692.

5. Haldane JS, Kellas AM and Kennaway EL. Experiments on acclimatization to reduced atmospheric pressure. J Physiol Cond 1919; 53:181.

6. Lutz BR and Schneider EC. Alveolar air and respiratory volume at low oxygen tensions. Am J Physiol 1919; 50: 280-301.

7. Gibson GE. Hypoxia. In: McCandless DW (ed) Cerebral energy metabolism and metabolic encephalopathy. New York: Plenum, 1985, pp. 43-78.

8. Castor M and Borgvall J. Technical report: the effects of mild, acute hypoxia on cognitive performance. Report No. 2015:20 2015. SSM Available at: www. stralsakerhetsmyndigheten.se.

9. McFarland RA. Stimuli primarily related to high altitude flight, in human factors in our transportation. Human Factors in Air Transportation, Occupational Health and Safety. New York: McGraw-Hill, 1953, pp. 153-169. 
10. Malle C, Quinette P, Laisney M, et al. Working memory impairment in pilots exposed to acute hypobaric hypoxia. Aviat Space Environ Med 2013; 84: 773-779.

11. Plum F and Posner JB. The diagnosis of stupor and coma. 3rd ed. Philadelphia: FA Davis Company, 1980.

12. Rumajogee P, Bregman T, Miller SP, et al. Rodent hypoxia-ischemia models for cerebral palsy research: a systematic review. Front Neurol 2016; 7: 57.

13. Zhang J and Piantadosi CA. Mitochondrial oxidative stress after carbon monoxide hypoxia in the rat brain. $J$ Clin Invest 1992; 90(4): 1193-1199.

14. Saligaut C, Moore N, Boulu R, et al. Hypobaric hypoxia: central catecholamine levels and cortical $\mathrm{PO}_{2}$ and avoidance response in rats treated with apomorphine. Aviat Space Environ Med 1981; 52(3): 166-170.

15. Groo D, Palosi E and Szporny L. Comparison of the effects of vinpocetine, vincamine, and nicergoline on the normal and hypoxia-damaged learning process in spontaneously hypertensive rats. Drug Dev Res 1988; 15(1): 75-85.

16. Shukitt-Hale B, Stillman MJ, Welch DI, et al. Hypobaric hypoxia impairs spatial memory in an elevation-dependent fashion. Behav Neural Biol 1994; 62: 244-252.

17. Shukitt-Hale B, Kadar T, Marlowe B, et al. Morphological alterations in the hippocampus following hypobaric hypoxia. Hum Exp Toxicol 1996; 15: 312-319.

18. Barhwal K, Singh SB, Hota SK, et al. Acetyl-L-carnitine ameliorates hypobaric hypoxic impairment and spatial memory deficits in rats. Eur J Pharmacol 2007; 570(1-3): 97-107.

19. Titus AD, Shankaranarayana Rao BS, Harsha HN, et al. Hypobaric hypoxia-induced dendritic atrophy of hippocampal neurons is associated with cognitive impairment in adult rats. Neurosci 2007; 145(1): 265-278.

20. Jayalakshmi K, Singh S, Kalpana B, et al. N-acetyl cysteine supplementation prevents impairment of spatial working memory functions in rats following exposure to hypobaric hypoxia. Physiol Behav 2007; 92: 643-650.

21. Hota SK, Barhwal K, Singh SB, et al. Chronic hypobaric hypoxia induced apoptosis in CA1 region of hippocampus: a possible role of NMDAR mediated p75 NTR upregulation. Exp Neurol 2008; 212: 5-13.

22. Hota SK, Barhwal K, Baithar I, et al. Bacopa monniera leaf extract ameliorates hypobaric hypoxia induced spatial memory impairment. Neurobiol Dis 2009; 34: 23-39.
23. Maiti P, Singh SB, Muthuraju S, et al. Hypobaric hypoxia damages the hippocampal pyramidal neurons in the rat brain. Brain Res 2007; 1175: 1-9.

24. Maiti P, Muthuraju S, Ilavazhagan G, et al. Hypobaric hypoxia induces dendritic plasticity in cortical and hippocampal pyramidal neurons in rat brain. Behav Brain Res 2008; 189: 233-243.

25. Maiti P, Singh SB and Ilavazhagan G. Nitric oxide system is involved in hypobaric hypoxia-induced oxidative stress in rat brain. Acta Histochem 2010; 112: 222-232.

26. Barhwal K, Hota SK, Baitharu I, et al. Isradipine antagonizes hypobaric hypoxia induced CA1 damage and memory impairment: complementary roles of L-type calcium channel and NMDA receptors. Neurobiol Dis 2009; 34: 230-244.

27. Barhwal K, Hota SK, Jain V, et al. Acetyl-1-carnitine (ALCAR) prevents hypobaric hypoxia-induced spatial memory impairment through extracellular related kinase-mediated nuclear factor erythroid 2-related factor 2 phosphorylation. Neurosci 2009; 161: 501-514.

28. Muthuraju S, Maiti P, Solanki P, et al. Acetylcholinesterase inhibitors enhance cognitive functions in rats following hypobaric hypoxia. Behav Brain Res, 2009; 203: 1-14.

29. Muthuraju S, Maiti P, Solanki P, et al. Cholinesterase inhibitors ameliorate spatial learning deficits in rats following hypobaric hypoxia. Exp Brain Res 2010; 203: 583-592.

30. Xu K, Sun X, Eroku BO, et al. Diet-induced ketosis improves cognitive performance in aged rats. $A d v \operatorname{Exp}$ Med Biol 2010; 662: 71-75.

31. Shi Q, Fu J, Ge D, et al. Huperzine A ameliorates cognitive deficits and oxidative stress in the hippocampus of rats exposed to acute hypobaric hypoxia. $\mathrm{Neu}$ rochem Res 2012; 37: 2042-2052.

32. Jain V, Baitharu I, Prasad D, et al. Enriched environment prevents hypobaric hypoxia induced memory impairment and neurodegeneration: role of BDNF/ PI3K/GSK $3 \beta$ pathway coupled with CREB activation. PloS One 2013; 8(5):e62235.

33. Prasad J, Baitharu I, Sharma AK, et al. Quercetin reverses hypobaric hypoxia-induced hippocampal neurodegeneration and improves memory function in the rat. High Altitude Medicine \& Biology 2013; 14: 383-394.

34. Baitharu I, Deep SN, Jain V, et al. Inhibition of glucocorticoid receptors ameliorates hypobaric hypoxia induced memory impairment in rat. Behav Brain Res 2013; 240: 76-86. 
35. Baitharu I, Jain V, Deep SN, et al. Withania somnifera root extract ameliorates hypobaric hypoxia induced memory impairment in rats. J Ethnopharmacol 2013; 145: 431-441.

36. Vornicescu C, Boşca B, Crişan D, et al. Neuroprotective effect of melatonin in experimentally induced hypobaric hypoxia. Rom J Morphol Embryol 2013; 54(4): 1097-1106.

37. Kauser H, Sahu S, Kumar S, et al. Guanfacine is an effective countermeasure for hypobaric hypoxia-induced cognitive decline. Neuroscience 2013; 254: 110-119.

38. Kauser H, Sahu S, Kumar S, et al. Guanfacine ameliorates hypobaric hypoxia induced spatial working memory deficits. Physiol Behav 2014; 123: 187-192.

39. Bert P. La pression barométrique: recherches de physiologie expérimentale. Masson Paris 1878; 1168.

40. Barcroft J. Respiratory function of the blood, Part I. New York: Cambridge University Press, 1925.

41. Savourey G, Launay JC, Besnard Y, et al. Normo- and hypobaric hypoxia: are there any physiological differences? Eur J Appl Physiol 2003; 89: 122-126.

42. Degache F, Larghi G, Faiss R, et al. Hypobaric versus normobaric hypoxia: same effects on postural stability? High Alt Med Biol 2012; 13: 40-45.

43. Neuhaus $\mathrm{C}$ and Hinkelbein J. Cognitive responses to hypobaric hypoxia: implications for aviation training Psychol Res Behav Manag 2014; 7: 297-302.

44. Shukitt-Hale B, Banderet LE and Lieberman HR. Elevation-dependent symptom, mood, and performance changes produced by exposure to hypobaric hypoxia. Int J Aviat Psychol 1998; 8: 319-334.

45. Du JY, Li XY, Zhuang Y, et al. Effects of acute mild and moderate hypoxia on human short memory. Space Med Eng (Beijing) 1999; 12: 270-273.

46. Virués-Ortega J, Buela-Casal G, Garrido E, et al. Neuropsychological functioning associated with high-altitude exposure. Neuropsychol Rev 2004; 14 : 197-224.

47. Rupp T, Jubeau M, Lamalle L, et al. Cerebral volumetric changes induced by prolonged hypoxic exposure and whole-body exercise. J Cereb Blood Flow Metab 2014; 34: 1802-1809.

48. McGuire SA, Sherman PM, Wijtenburg SA, et al. White matter hyperintensities and hypobaric exposure. Ann Neurol 2014; 76: 719-726.

49. Garrido E, Segura R, Capdevila A, et al. New evidence from magnetic resonance imaging of brain changes after climbs at extreme altitude. Eur J Appl Physiol Occup Physiol 1995; 70: 477-481.
50. Paola MD, Bozzali M, Fadda L, et al. Reduced oxygen due to high-altitude exposure relates to atrophy in motor-function brain areas. Eur J Neurol 2008; 15: 1050-1057.

51. Foster GE, Davies-Thompson J, Dominelli PB, et al. Changes in cerebral vascular reactivity and structure following prolonged exposure to high altitude in humans. Physiol Rep 2015; 3: e12647.

52. Schommer K, Kallenberg K, Lutz K, et al. Hemosiderin deposition in the brain as footprint of high-altitude cerebral edema. Neurology 2013; 81: 1776-1779.

53. Fayed N, Modrego PJ and Morales H. Evidence of brain damage after high-altitude climbing by means of magnetic resonance imaging. Am J Med 2006; 119(2): 168.e1-6.

54. Pulsinelli WA. Selective neuronal vulnerability: morphological and molecular characteristics. Prog Brain Res 1985; 63: 29-37.

55. Hurn PD, Vannucci SJ and Hagberg H. Adult or perinatal brain injury does sex matter? Stroke 2005; 36: 193-195.

56. Mayoral SR, Omar G and Penn AA. Sex differences in a hypoxia model of preterm brain damage. Pediatr Res 2009; 66: 248-253.

57. Gibson GE and Huang HM. Animal models of brain hypoxia. In: Boulton AA, Baker GB and Roger F (eds) Animal models of neurological disease. Totowa NJ: The Humana Press Inc, 1992, pp. 51-93.

58. McCarty R and Kopin IJ. Patterns of behavioral development in spontaneously hypertensive rats to inescapable footshock. Dev Psychobiol 1979; 12: 239-243.

59. Krauchi K, Witz-Justice A, Willener R, et al. Spontaneous hypertensive rats: behavioral and corticosterone response depend on circadian phase. Physiol Behav 1983; 30: 35-40.

60. Rybnikova E, Sitnik N, Gluschenko T, et al. The preconditioning modified neuronal expression of apoptosis-related proteins of Bcl-2 superfamily following severe hypobaric hypoxia in rats. Brain Res 2006; 1089(1): 195-202.

61. Hota KB, Hota SK, Srivastava RB, et al. Neuroglobin regulates hypoxic response of neuronal cells through Hif- $1 \alpha$ - and Nrf2-mediated mechanism. J Cereb Blood Flow Metab 2012; 32(6): 1046-1060.

62. Chiş IC, Baltaru D, Dumitrovici A, et al. Quercetin ameliorate oxidative/nitrosative stress in the brain of rats exposed to intermittent hypobaric hypoxia. Rev Virtual Quim 2016; 8(2): 369-383.

63. Liu J, Head E, Gharib AM, et al. Memory loss in old rats is associated with brain mitochondrial decay and 
RNA/DNA oxidation; partial reversal by feeding acetyl-1-carnitine and/or R-alpha lipoic acid. Proc Natl Acad Sci 2002; 99(4): 2356-2361.

64. Martilla RJ, Roytta M, Lorentz H, et al. Oxygen toxicity protecting enzymes in the human brain. $J$ Neural Transm 1988; 74: 87-90.

65. Watson DB. Effect of concomitance of lipid peroxidation in experimental models of cerebral ischemia and stroke. Prog Brain Res 1996; 96: 69-90.

66. Li RC. Nitric oxide synthase and intermittent hypoxia-induced spatial learning deficit in the rat. Neurobiol Dis 2004; 17: 44-53.

67. Matsui T, Nagafuji T, Kumanishi T, et al. Role of nitric oxide pathogenesis underlying ischemic cerebral damage. Cell Mol Neurobiol 1999; 19: 177.

68. Welsh SJ, Bellamy WT, Briehl MM, et al. The redox protein thioredoxin-1 (Trx-1) increases hypoxia-inducible factor 1alpha protein expression: Trx-1 overexpression results in increased vascular endothelial growth factor production and enhanced tumor angiogenesis. Cancer Res 2002; 62(17): 5089-5095.

69. Chandel NS, Maltepe E, Goldwasser E, et al. Mitchondrial reactive species triggerhypoxia induced transcription. Proc Natl Acad Sci USA 1998; 95: 11715-11720.

70. Kaluz S, Kaluzova M and Stanbridge EJ. Regulation of gene expression by hypoxia: integration of the HIF-transduced hypoxic signal at the hypoxia-responsive element. Clin Chim Acta 2008; 395(1-2): 6-13.

71. Romero RM, Canuelo A, Siles E, et al. Nitric oxide modulates hypoxia-inducible factor- 1 and poly (ADPribose) polymerase-1 cross talk in response to hypobaric hypoxia. J Appl Physiol 2012; 112(5): 816-823.

72. McCord JM. The evolution of free radicals and oxidative stress. Am J Med 2000; 198: 652.

73. Nguyen T, Sherratt PJ, Nioi P, et al. Nrf2 controls constitutive and inducible expression of ARE-driven genes through a dynamic pathway involving nucleocytoplasmic shuttling by Keap1. J Biol Chem 2005; 280: 32485-32492.

74. Saido TC, Nagao S, Shiramine M, et al. Distinct kinetics of subunit autolysis in mammalian m-calpain activation. FEBS Lett 1994; 346: 263-267.

75. Villa PG, Henzel WJ, Sensenbrenner M, et al. Calpain inhibitors, but not caspase inhibitors, prevent actin proteolysis and DNA fragmentation during apoptosis. $J$ Cell Sci 1998; 111: 713-722.

76. Lankiewicz S, Luetjens MC, Bui TN, et al. Activation of calpain I converts excitotoxic neuron death into a caspase-independent cell death. J Biol Chem 2000; 275: 17064-17071.

77. Gorman AM, Ceccatelli S and Orrenius S. Role of mitochondria in neuronal apoptosis. Dev Neurosci 2000; 22: 348-358.

78. Kroemer G and Reed JC. Mitochondrial control of cell death. Nat Med 2000; 6: 513-519.

79. Nicholls D and Attwell D. The release and uptake of excitatory amino acids. Trends Pharmacol Sci 1990; 11: 462-468.

80. Hota SK, Hota BK, Prasad D, et al. Oxidative-stress-induced alterations in Sp factors mediate transcriptional regulation of the NR1 subunit in hippocampus during hypoxia. Free Radic Biol Med 2010; 49: 178-191.

81. Sattler R and Tymianski M. Molecular mechanisms of calcium dependent excitotoxicity. J Mol Med 2000; 78: 3-13.

82. Dugan LL, Sensi SL, Canzoniero LM, et al. Mitochondrial production of reactive oxygen species in cortical neurons following exposure to N-methyl-D-aspartate. J Neurosci 1995; 15: 6377-6388.

83. Reynolds IJ and Hastings TG. Glutamate induces the production of reactive oxygen species in cultured forebrain neurons following NMDA receptor activation. $J$ Neurosci 1995; 15: 3318-3327.

84. Gunter TE, Buntinas L, Sparagna GC, et al. The $\mathrm{Ca}^{2+}$ transport mechanisms of mitochondria and $\mathrm{Ca}^{2+}$ uptake from physiological type $\mathrm{Ca}^{2+}$ transients. Biochim Biophys Acta 1998; 1366: 5-15.

85. Stout AK, Raphael HM, Kanterewicz BI, et al. Glutamate induced neuron death requires mitochondrial calcium uptake. Nat Neurosci 1998; 1: 366-373.

86. Luetjens CM, Bui NT, Sengpiel B, et al. Delayed mitochondrial dysfunction in excitotoxic neuron death: cytochrome $\mathrm{c}$ release and a secondary increase in superoxide production. $J$ Neurosci 2000; 20: 5715-5723.

87. Sengpiel B, Preis E, Krieglstein J, et al. NMDA-induced superoxide production and neurotoxicity in cultured rat hippocampal neurons: role of mitochondria. Eur J Neurosci 1998; 10: 1903-1910.

88. Carriedo SG, Sensi SL, Yin HZ, et al. AMPA exposures induce mitochondrial $\mathrm{Ca}^{+}$overload and ROS generation in spinal motor neurons in vitro. $J$ Neurosci 2000; 20: 240-250.

89. Kluck RM, Bossy-Wetzel E, Green DR, et al. The release of cytochrome $\mathrm{c}$ from mitochondria: a primary site for Bcl-2 regulation of apoptosis. Science 1997; 275: 1132-1136.

90. Green DR and Reed JC. Mitochondria and apoptosis. Science 1998; 281: 1309-1312. 
91. Shimizu S, Narita M and Tsujimoto Y. Bcl-2 family proteins regulate the release of apoptogenic cytochrome $\mathrm{c}$ by the mitochondrial channel VDAC. Nature 1999; 399: 483-487.

92. Tsai FS, Peng WH, Wang WH, et al. Effects of luteolin on learning acquisition in rats: involvement of the central cholinergic system. Life Sci 2007; 80: 1692-1698.

93. Gericke CA, Lang UE, Steckler T, et al. Nerve growth factor response to excitotoxic lesion of the cholinergic basal forebrain is slightly impaired in aged rats. $J$ Neural Transm 2003; 110: 627-639.

94. Arneric SP and Wiliams M. Nicotinic agonists in Alzheimer's disease: does the molecular diversity of nicotine receptors offer the opportunity for developing CNS selective cholinergic channel activators? In: Racagni G, Brunello N and Langer SZ (eds) Recent advances in the treatment of neurodegenerative disorders and cognitive dysfunction. Basel: Darger, 1994, pp. 58-70.

95. Muthuraju S, Maiti P, Solanki P, et al. Possible role of cholinesterase inhibitors on memory consolidation following hypobaric hypoxia of rats. Int $J$ Neurosci 2011; 121: 279-288.

96. McAllister AK. Neurotrophins and neuronal differentiation in the central nervous system. Cell Mol Life Sci 2001; 58: 1054-1060.

97. Dreyfus CF. Effects of nerve growth factor on cholinergic brain neurons. Trends Pharmacol Sci 1989, 10: 145-149.

98. Goldman-Rakic PS. Cellular basis of working memory. Neuron 1995; 14: 477-485.

99. Arnsten AFT. Through the looking glass: differential noradrenergic modulation of prefrontal cortical function. Neural Plast 2000; 7: 1-2.

100. Sakamoto K, Karelina K and Obrietan K. CREB: a multifaceted regulator of neuronal plasticity and protection. $J$ Neurochem 2011; 116: 1-9.

101. Bonni A, Brunet A, West AE, et al. Cell survival promoted by the Ras-MAPK signaling pathway by transcription-dependent and independent mechanisms. Science 1999; 286: 1358-1362.

102. Du K and Montminy M. CREB is a regulatory target for the protein kinase AKT/PKB. J Biol Chem 1998; 273: 32377-32379. 